\title{
Acetaldehyde Adducts in Blood and Bone Marrow of Patients With Ethanol-Induced Erythrocyte Abnormalities
}

\author{
Jaana Latvala, ${ }^{1,2}$ Seppo Parkkila, ${ }^{2,3}$ Jukka Melkko, $^{4}$ and Onni Niemelä ${ }^{1,2}$ \\ ${ }^{1}$ EP Central Hospital Laboratory, Seinäjoki, Finland \\ ${ }^{2}$ Department of Clinical Chemistry, University of Oulu, Oulu, Finland \\ ${ }^{3}$ Department of Anatomy and Cell Biology, University of Oulu, Oulu, Finland \\ ${ }^{4}$ Department of Pathology, University of Oulu, Oulu, Finland \\ Accepted February 15, 2001
}

\begin{abstract}
Background: Although alcohol abuse is known to cause a wide array of adverse effects on blood cell formation, the molecular mechanisms by which alcohol exerts its toxic actions remain poorly defined. We examine here the formation of acetaldehyde-derived protein modifications in erythrocytes and in their bone marrow precursors using antibodies specifically recognizing acetaldehyde-modified epitopes in proteins independently of the nature of the carrier protein.

Materials and Methods: We studied 138 consecutive adult patients undergoing bone marrow aspiration due to macrocytosis (MCV values above $99 \mathrm{fL}$ ). Assessment included complete blood counts, morphologic review, assessment of alcohol consumption, and biochemical and immunocytochemical assays for acetaldehyde adducts.

Results: There were 68 patients $(49 \%)$ with a history of excessive alcohol consumption, $28(20 \%)$ of whom were patients with severe dependence. The blood smears prepared from the alcoholic patients with macrocytosis also
\end{abstract}

contained stomatocytes and knizocytes. Bone marrow aspirates from 12 alcoholic patients showed vacuolization of pronormoblasts and the presence of ring sideroblasts was noted in 8 cases. In immunocytochemical analyses of the peripheral blood erythrocytes, acetaldehyde-derived epitopes were found to occur both on the cell membrane and inside the erythrocytes. Bone marrow aspirates also showed positive staining for acetaldehyde adducts in the erythropoietic cells in 8 of $11(73 \%)$ consecutive alcoholic patients. Separation of the erythrocyte proteins from the samples of alcoholics on HPLC-chromatography revealed the formation of fast-eluting hemoglobin fractions, which also reacted with antibodies against acetaldehyde adducts. Conclusions: Current data suggest that acetaldehydeerythrocyte adducts are formed in vivo in blood and bone marrow of patients with excessive alcohol consumption. This may contribute to the generation of the erythrocyte abnormalities, which are frequently observed in alcoholic patients.

\section{Introduction}

Excessive ethanol intake is associated with a wide variety of morphologic abnormalities both in blood cells and in their bone marrow progenitors (1). The mechanisms by which such effects are brought about, however, remain unclear.

On blood smears, erythrocytes from alcoholics frequently show round macrocytosis and other morphologic aberrations. In bone marrow, vacuolized erythroblasts and sideroblastic alterations may be seen (1). Although some of the effects of ethanol are associated with a concomitant folate deficiency or with the presence of underlying liver disease, a direct toxic effect of ethanol has also been postulated as a mechanism of ethanol toxicity.

Recent studies have indicated that acetaldehyde, the first metabolite of ethanol, may be responsible for a variety of adverse effects of ethanol (2-5). High

Address correspondence and reprint requests to: Onni Niemelä, MD, EP Central Hospital Laboratory, FIN-60220 Seinäjoki, Finland. Phone: 1 358-6-415 4719; Telefax: 1 358-6-415 4924; E-mail: onni.niemela@epshp.fi concentrations of acetaldehyde have been found to occur inside the erythrocytes of alcoholics, which may suggest a role for erythrocytes as bioreactors to remove acetaldehyde formed upon ethanol consumption (6-8). A number of previous in vitro studies have further shown that acetaldehyde is able to bind and form covalent adducts with proteins and cellular constituents (9-11), including hemoglobin protein (12) and erythrocyte membranes (13). Several studies in both experimental animals and human alcoholics have also demonstrated acetaldehydeprotein adduct formation with liver proteins in vivo $(4,14,15)$.

The present study was designed to examine whether acetaldehyde-erythrocyte protein adducts are generated in vivo in blood and bone marrow of patients with macrocytosis. Ethanol consumption was found to be a common cause of erythrocyte abnormalities. The presence of acetaldehyde-erythrocyte adducts in blood smears and bone marrow of such patients is consistent with the view that acetaldehyde plays a role in creating the toxic effects of ethanol on erythropoiesis. 


\section{Materials and Methods}

\section{Patients}

In a total of 820 consecutive patients undergoing bone marrow examination at Southern Osthrobothnia Central Hospital, Seinäjoki, Finland, there were 138 patients (85 males, 53 females) with elevated mean corpuscular volume who were included in the study. Assessment included complete blood counts, morphologic review, assessment of alcohol consumption, measurements of liver enzymes, erythrocyte folate, serum vitamin $B_{12}$, and biochemical and immunocytochemical assays for acetaldehyde adducts. Ethanol consumption was examined in connection with the clinical assessment by detailed questions on the use of alcoholic beverages. In this sample, there were $68(49 \%)$ patients (45 males and 23 females, mean age 51 years) with a history of excessive alcohol consumption (ethanol consumption exceeding a mean of $60 \mathrm{~g} / \mathrm{day}$ of ethanol either continuously or during repeated inebriations). There were 28 patients (23 males, 5 females, mean age 54 years) who met the DSM-IV criteria for severe alcohol dependence on the grounds of their case history and clinical examination, and who had been actively drinking during the period of 1-2 weeks prior to sampling in amounts exceeding a mean of $120 \mathrm{~g} /$ day. The time of abstinence prior to sampling varied between 0 and 3 days. Blood smears and bone marrow aspirates from all patients were prepared for routine diagnostic purposes. Erythrocyte proteins were prepared from EDTA-anticoagulated blood samples, as described below. In addition, blood smears and samples from bone marrow aspirates were also used for immunologic and immunocytochemical analyses of acetaldehyde-derived protein adducts from 11 alcoholic and 9 nondrinking patients with macrocytosis.

The procedure was approved by the institutional review board and the study was carried out according to the provisions of the Declaration of Helsinki.

\section{Preparation of Erythrocyte Proteins and Acetaldehyde Labeling In Vitro}

The erythrocytes from EDTA-anticoagulated blood samples were separated by centrifugation and washed three times with phosphate-buffered saline (PBS), pH 7.4. For acetaldehyde labelling in vitro, aliquots of the erythrocyte protein from the nondrinking controls were first hemolyzed with 20 volumes of polyoxyethylene ether, $0.1 \% \mathrm{v} / \mathrm{v}$ in borate buffer (Hemolysis Reagent, DIAMAT Analyzer System, Bio-Rad, Hercules, CA, USA) and incubated for $35 \mathrm{~min}$ at $378 \mathrm{C}$ to remove the unstable Schiff bases. The hemolysates were dialyzed twice against PBS. Thereafter, $1 \mathrm{ml}$ of the hemolysate was incubated for $3 \mathrm{hr}$ at room temperature with various concentrations of ${ }^{14} \mathrm{C}$-acetaldehyde-solution in PBS including $10 \mu \mathrm{l}$ of $1 \mathrm{M} \mathrm{NaCNBH}$ as a reducing agent. The reaction mixture was subsequently dialyzed against PBS. The labeled proteins were stored at $2708 \mathrm{C}$ until used.

\section{HPLC Separation and Immunologic Assays}

The erythrocyte proteins were separated into subfractions using HPLC, as previously described (16). The column was a cation exchanger, Mono S HR5/5 (Pharmacia Fine Chemicals, Uppsala, Sweden), and lithium was used as the elution cation. The flow rate was $2.0 \mathrm{ml} / \mathrm{min}$, and $0.5 \mathrm{ml}$ fractions were collected. Hemoglobin fractions were monitored at $405 \mathrm{~nm}$. All fractions were subsequently tested for immunologic reactivity by ELISA using previously characterized rabbit, polyclonal, affinity-purified antibodies against acetaldehyde adducts $(10,14)$. These were raised against BSA conjugated with $1 \mathrm{mM}$ acetaldehyde under reducing conditions (14). The antibodies reacted with acetaldehyde-protein condensates prepared at $5 \mu \mathrm{M}$ to $10 \mathrm{mM}$ concentrations of acetaldehyde independent of the nature of the carrier protein. Samples of the HPLC fractions were first adsorbed on cavities of microtiter plates (Nunc Maxisorb, Roskilde, Denmark). After treatment with gelatin, the rabbit anti-acetaldehydeadduct antiserum (1:200 in PBS-Tween) was added and incubated for $\mathrm{l} \mathrm{hr}$ at $378 \mathrm{C}$. After extensive washes, goat anti-rabbit immunoglobulin labeled with alkaline phosphatase was incubated on plates over night at $48 \mathrm{C}$. P-nitrophenylphosphate solution was used as a color reaction substrate (Alkaline Phosphatase Substrate Kit, Bio-Rad Laboratories, Hercules, CA, USA). The color reaction was stopped by adding $100 \mu \mathrm{l}$ of $0.4 \mathrm{M} \mathrm{NaOH}$. The intensities of the reactions were measured by Anthos HtII microplate reader (Anthos Labtec Instruments, Salzburg, Austria).

\section{Immunocytochemical Methods}

Immunocytochemical analyses for the acetaldehyde adducts from blood smears and bone marrow aspirates were carried out from 20 patients (11 alcoholic and 9 control patients) blind to the knowledge of the clinical data. Isolated peripheral blood cells were spread onto the microscope slides and fixed in $4 \%$ formaldehyde for $20 \mathrm{~min}$. Saponin $(0.05 \%)$ was used to permeabilize the cells. The steps in the immunofluorescence staining of the cell preparations were essentially the same as described by Parkkila et al. (17). For confocal laser scanning microscopy, the samples were excited with a laser beam at a wavelength of $568 \mathrm{~nm}$ using an air-cooled argonkrypton laser (75 mW output; Leitz CLSM, Leica Laser Technics, Wetzlar, Germany). The emission light was focused through a pinhole aperture and the full field was scanned in square image formats of 5123512 pixels. Built-in software was used to reconstruct the images obtained from the confocal sections. Digital image analysis was carried out with a Nikon Diaphot-TMD microscope (Nikon, Tokyo, Japan) combined with a Dage model 72E CCD camera with remote control (Dage-MTI, Michigan City, IN, USA) and an MCID-M2 image analyzer (Imaging 
Research, St. Catharines, Ontario, Canada) with a spatial resolution of 128031024 pixels (18).

\section{Other Methods}

Blood cell counts were determined using Coulter, Miami, FL, USA, STKS Hematology Analyzer. Measurements of aspartate aminotransferase, alanine aminotransferase, erythrocyte folate, and serum vitamin $B_{12}$ levels were carried out using standard clinical chemical methods.

\section{Results}

In a sample of 138 consecutive hospitalized patients or outpatients with macrocytosis undergoing bone marrow aspiration or biopsy, there were 68 patients $(49 \%)$ with a history of excessive alcohol consumption, 28 of whom were patients with severe dependence. The degree of macrocytosis in the alcoholconsuming patients showed a slight correlation with the amount of self reported ethanol intake $\left(r_{s} 50.26\right.$, $p, 0.05)$. There were 5 patients with liver fibrosis or cirrhosis; the remaining alcohol-consuming patients were devoid of any clinical or biochemical signs of significant liver disease. In the group of alcohol abusers, 15 patients had anemia, 6 of whom also had low erythrocyte folate levels.

Bone marrow aspirates from the alcoholic patients showed excessive vacuolization of pronormoblasts in 12 patients, all of whom were patients with severe alcohol dependence and recent intoxication (Fig. 1). Abnormal sideroblasts were found in 8 patients. Samples of bone marrow from 11 consecutive alcoholic patients and from 9 nonalcoholic controls were processed for immunocytochemical stainings for acetaldehyde-modified epitopes (Fig. 2). Positive staining was found in the late (nonnucleated) erythropoietic cells in $8(73 \%)$ of the alcoholic patients (Fig. 2A-D), whereas no specific staining was seen in the bone marrow cells of control patients (Fig. 2F). The erythrocyte cell membranes were found to be the primary sites of adduct

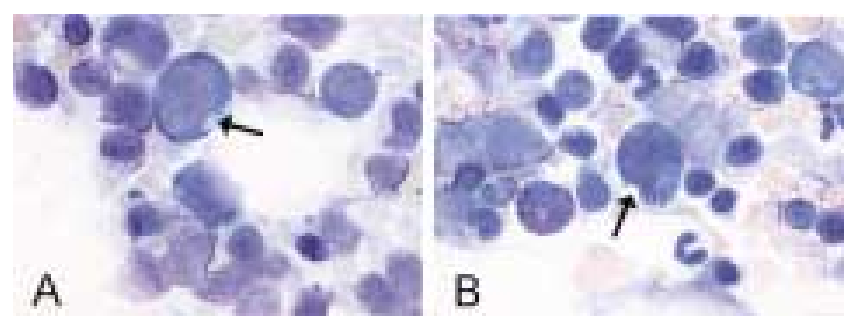

Fig. 1. Photographs of bone marrow from alcoholics. In a sample of 138 consecutive patients undergoing bone marrow aspiration due to macrocytosis, 68 patients $(49 \%)$ had a history of excessive alcohol consumption, 28 of whom were patients with severe ethanol dependence. Bone marrow aspirates from 12 alcoholic patients showed vacuolization of pronormoblasts (A,B). May-Grunwald-Giemsa stains of 69-year-old male alcoholic patient (A) and 39-year-old female patient with ethanol dependence (B) are shown.
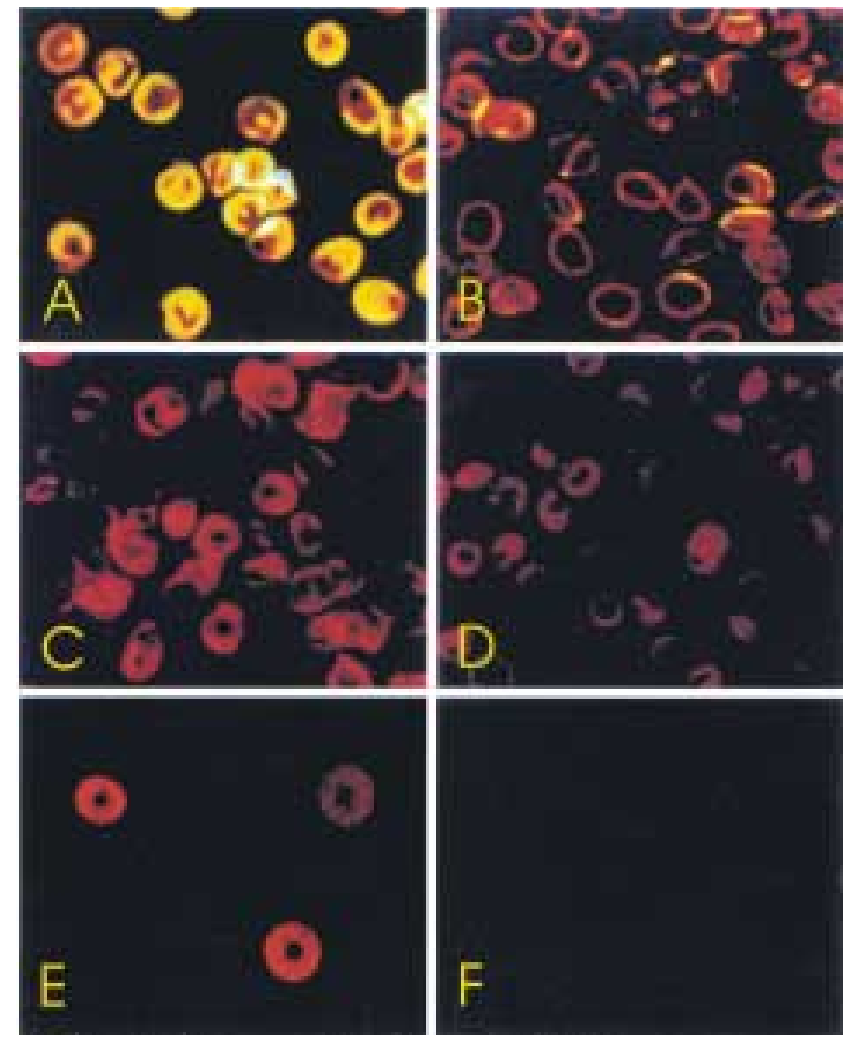

Fig. 2. Immunocytochemical stainings for acetaldehyde adducts. Confocal fluorescence micrographs of bone marrow specimens (A-D,F) and peripheral blood erythrocytes (E), which were stained immunocytochemically for the presence of acetaldehyde adducts from 11 samples representing consecutive alcoholic patients and from 9 nonalcoholic patients with macrocytosis. The stainings were carried out with monospecific antibodies recognizing acetaldehyde-derived epitopes in proteins independent of the nature of the carrier protein. Acetaldehyde adducts in the erythropoietic cells were found in 8 of $11(73 \%)$ of the alcohol-abusing patients. Figures A-D demonstrate the staining data from four different individual patients. Positive staining also occurred in the circulating erythrocytes both inside the erythrocytes and on the cell membrane (E). Routine examination of the blood smears prepared from the alcoholic patients also revealed the presence of stomatocytes and knizocytes. Stainings of bone marrow samples from nonalcoholic patients were devoid of specific signal (F).

deposition (Fig. 2B,C). The early erythroblasts and other marrow cell lineages were usually devoid of specific staining.

In this sample, only one alcoholic patient showed a positive reaction in the blastic cells. The peripheral red blood cell morphology from the chronic alcoholic patients showed the presence of round macrocytes, stomatocytes, and knizocytes. In immunocytochemical analyses, the blood smears prepared from the alcohol abusers were also found to contain acetaldehyde-derived epitopes both on the cell membrane and inside the erythrocytes (Fig. 2E). Although membrane adducts were present in all of the immunopositive alcoholic patients, intracellular adducts occurred in $60 \%$ of such patients. The pa- 

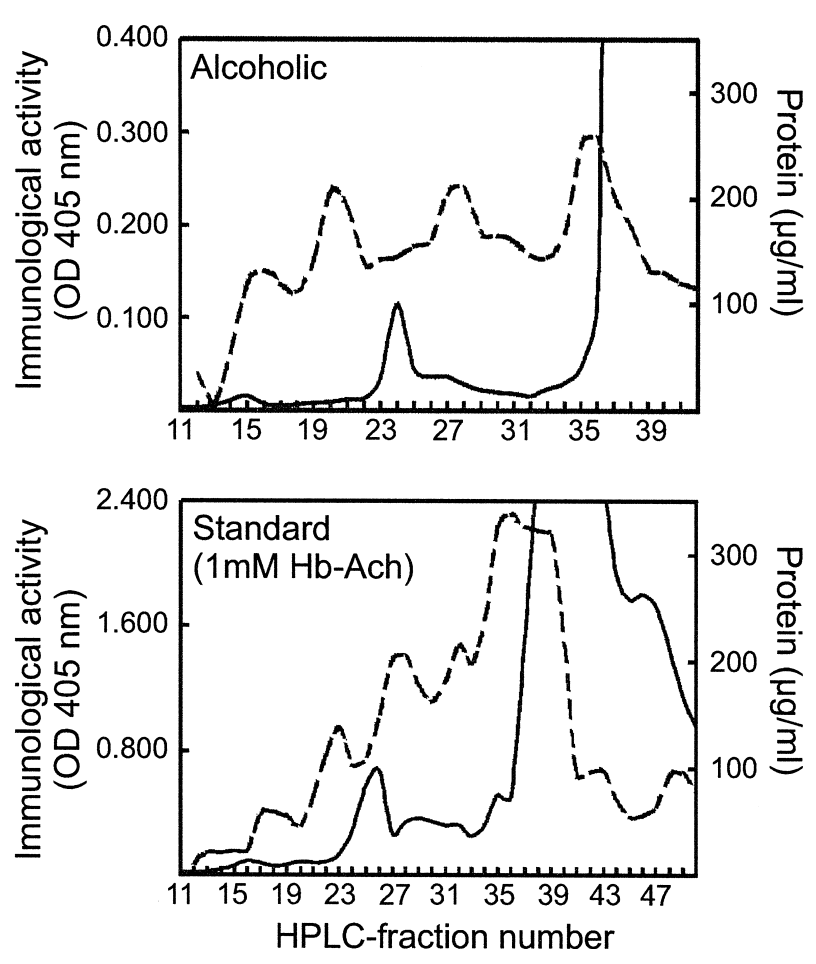

Fig. 3. HPLC chromotographs of acetaldehyde-modified erythrocycle proteins. Upper panel: Separation of the erythrocyte proteins on HPLC chromatography from the samples of the alcoholics revealed the formation of fast-eluting hemoglobin fractions that reacted with the antibodies against the acetaldehyde-derived epitopes in proteins. Lower panel: Similar sites of immunologic activity also occurred in erythrocyte proteins prepared in vitro with incubation of erythrocyte proteins and $1 \mathrm{mM}$ concentration of acetaldehyde. Immunologic activity was monitored using antibodies against acetaldehyde adducts.

Dashed line, immunologic activity $\left(\mathrm{OD}_{405} \mathrm{~nm}\right)$. Solid line, concentration of total protein $(\mu \mathrm{g} / \mathrm{ml})$.

tients exhibiting positive staining in bone marrow also had immunopositive erythrocytes in circulation. However, the degree of macrocytosis within the group of alcoholics with elevated mean corpuscular volume was not found to correlate significantly with amount of erythrocyte adducts $\left(r_{s} 50.15\right)$, as assessed using confocal laser scanning microscopy and digital image analyses of the staining data.

Separation of the erythrocyte proteins from the blood samples of the alcoholics on HPLC chromatography revealed the formation of fast-eluting hemoglobin fractions, which reacted with the antibodies against the acetaldehyde adducts. The HPLC-profiles showed three main peaks of immunologic activity occurring at similar sites as those of the erythrocyte proteins labeled in vitro with acetaldehyde (Fig. 3).

\section{Discussion}

To our knowledge, the present data are the first demonstration that acetaldehyde, the first metabolite of ethanol, is capable of forming stable adducts with erythroid proteins and cellular constituents in bone marrow in vivo. The present findings are con- sistent with a number of previous studies that demonstrated the capability of acetaldehyde to form adducts with intracellular proteins and various cell types $(5,6,8-13)$. Increasing evidence has also accumulated on the formation of protein adducts in vivo with acetaldehyde and liver proteins as a result of ethanol oxidation $(4,5,14,15)$. As a consequence, this may interfere with protein function $(19,20)$, stimulate immunologic responses $(3,20,21)$, and shorten the biological half-life of the modified proteins (22). Apparently, acetaldehyde-modified erythrocytes may also be more vulnerable to damage, hemolysis, and shortened biological half-life in vivo (22).

Current data indicate that acetaldehyde may play a role in the hematologic derangements in alcoholic patients, who are known to present distinct morphologic aberrations both in bone marrow and peripheral blood (1). The occurrence of acetaldehyde-derived epitopes in peripheral blood erythrocytes and in their bone marrow precursors supports a direct role of ethanol metabolites in creating hematotoxicity, which is in accordance with several lines of previous observations (1,23-25). After heavy alcohol intake, abstinence is usually followed by reticulocytosis. A cytotoxic effect of ethanol is also supported by the occurrence of erythroblast vacuolization in bone marrow, as seen here in approximately one-third of the alcohol-dependent patients in the absence of any other bone marrow toxic agent. Such vacuoles usually disappear with abstinence $(1,23)$. Interestingly, the early blastic cells were, however, usually devoid of specific staining for acetaldehyde adducts, suggesting a lack of apparent correlation between immunocytochemically detectable acetaldehyde adduct deposition and erythroblast vacuolization.

Previous in vitro studies have shown that ethanol and acetaldehyde may inhibit colony formation by committed stem cells, the erythroid cells being particularly vulnerable to ethanol action (24). Alcohol abuse is also known to alter the lipid structural order in human erythrocyte membranes (26). Wickramasinghe and Malik (27) reported that even relatively low concentrations of acetaldehyde, although not ethanol, can cause an impairment of cell proliferation and an abnormality of cell growth in vitro, supporting a specific role of acetaldehyde in increasing the mean cell volume of erythrocytes. Although the reason why the marrow specimens from some alcoholic patients stained positively and others did not is still unclear, it may be speculated that the time of abstinence prior to sampling, the amount of alcohol intake, and different individual susceptibilities may all play a role. Acetaldehyde-derived erythrocyte modifications appear to be most abundant at the late stages of erythrocyte development. No apparent correlations existed, however, between the amount of self-reported ethanol intake and marrow positivity or between the degree of macrocytosis and marrow positivity within the group of alcoholics with elevated mean corpuscular volume. 
Although ethanol consumption seems to be an increasingly common cause of macrocytosis in general hospitals (28-30), most of such patients tend to escape detection. Nevertheless, elevated mean corpuscular volume has frequently been used as a screening tool for alcohol abuse despite the fact that it has a relatively low sensitivity for this purpose. Based on the present findings, future work appears warranted to examine the possibility that immunologic and chromatographic assays for acetaldehydederived protein modifications from peripheral blood erythrocytes and bone marrow could be used in the differential diagnosis of alcohol abuse as an underlying cause of hematologic symptoms. Such assays should perhaps be targeted to erythrocyte membrane proteins or to the hemoglobin fractions enriched by chromatographic techniques instead of total hemoglobin $(12,16,22,31-33)$. Because thrombopoiesis is also known to be affected by ethanol, future studies should address the possibility whether acetaldehyde adducts are also present in platelet protein lysates and whether acetaldehyde could play a role in the effects of ethanol on blood platelet metabolism.

\section{Acknowledgments}

The present studies were supported by the Finnish Foundation for Alcohol Studies. The expert technical assistance of Aki Pulkkinen in the immunocytochemical analyses is gratefully acknowledged.

\section{References}

1. Lindenbaum J. (1987) Hematologic complications of alcohol abuse. Semin. Liver Dis. 7: 169-181.

2. Lieber CS. (1995) Medical disorders of alcoholism. N. Engl. J. Med. 333: 1058-1065.

3. Tuma DJ, Klassen LW. (1992) Immune responses to acetaldehyde-protein adducts: role in alcoholic liver disease. Gastroenterology 103: 1969-1973.

4. Niemelä O, Parkkila S, Ylä-Herttuala S, Villanueva J, Ruebner B, Halsted C. (1995) Sequential acetaldehyde production, lipid peroxidation, and fibrogenesis in micropig model of alcohol induced liver disease. Hepatology 22: 1208-1214.

5. Lin RC, Dai J, Lumeng L, Zhang M. (1995) Serum low density lipoprotein of alcoholic patients is chemically modified in vivo and induces apolipoprotein $\mathrm{E}$ synthesis by macrophages. J. Clin. Invest. 95: 1979-1986.

6. Hernández-Muños R, Baraona E, Blacksberg I, Lieber CS. (1989) Characterization of the increased binding of acetaldehyde to red blood cells in alcoholics. Alcohol Clin. Exp. Res. 13: 654-659.

7. Magnani M, Laguerre M, Rossi L, et al. (1989) Acetaldehyde dehydrogenase-loaded erythrocytes as bioreactors for the removal of blood acetaldehyde. Alcohol Clin. Exp. Res. 13: 849.

8. Peterson CM, Jovanovic- Peterson L, Schmid-Formby F. (1988) Rapid association of acetaldehyde with hemoglobin in human volunteers after low dose ethanol. Alcohol 5: 371-374.

9. Smith SL, Jennett RB, Sorrell MF, Tuma DJ. (1989) Acetaldehyde substoichiometrically inhibits bovine neurotubulin polymerization. J. Clin. Invest. 84: 337-341.

10. Israel Y, Hurwitz E, Niemelä O, Arnon R. (1986) Monoclonal and polyclonal antibodies against acetaldehyde-containing epitopes in acetaldehyde-protein adducts. Proc. Natl. Acad. Sci. U.S.A. 83: 7923-7927.

11. Trudell JR, Ardies CM, Anderson WR. (1991) The effect of alcohol and anesthetic metabolites on cell membranes. A pos- sible direct immune mechanism. Ann. N Y Acad. Sci. 625: 806-817.

12. Stevens VJ, Fantl WJ, Newman CB, Sims RV, Cerami A, Peterson CM. (1981) Acetaldehyde adducts with hemoglobin. J. Clin. Invest. 67: 361-369.

13. Gaines KC, Salhany JM, Tuma DJ, Sorrell MF. (1979) Reaction of acetaldehyde with human erythrocyte membrane proteins. FEBS Lett. 75: 115-119.

14. Niemelä O, Juvonen T, Parkkila S. (1991) Immunohistochemical demonstration of acetaldehyde modified epitopes in human liver after alcohol consumption. J. Clin. Invest. 87: 1367-1374.

15. Holstege A, Bedossa P, Poynard T, et al. (1994) Acetaldehydemodified epitopes in liver biopsy specimens of alcoholic and non-alcoholic patients: localization and association with progression of liver fibrosis. Hepatology 19: 367-374.

16. Sillanaukee $P$, Seppä $K$, Koivula $T$, Israel $Y$, Niemelä $O$. (1992) Acetaldehyde-modified hemoglobin as a marker of alcohol consumption: comparison of two new methods. J. Lab. Clin. Med. 120: 42-47.

17. Parkkila S, Rajaniemi H, Parkkila AK, et al. (2000) Carbonic anhydrase inhibitor suppresses invasion of renal cancer cells in vitro. Proc. Natl. Acad. Sci. U.S.A. 97: 2220-2224.

18. Parkkila S, Niemelä O, Britton RS, et al. (1996) Vitamin E decreases hepatic levels of aldehyde-derived peroxidation products in rats with iron overload. Am. J. Physiol. 270: G376-G384.

19. Mauch TJ, Donohue TM, Zettermann RK, Sorrell MF, Tuma DJ. (1986) Covalent binding of acetaldehyde selectively inhibits the catalytic activity of lysine dependent enzymes. Hepatology 6: 263-269.

20. Nicholls R, de Jersey J, Worrall S, Wilce P. (1992) Modification of proteins and other biological molecules by acetaldehyde: adduct structure and functional significance. Int. J. Biochem. 24: 1899-1906.

21. Viitala K, Blake JE, Israel Y, Niemelä O. (1997) Serum IgA, IgG, and IgM antibodies directed against acetaldehydederived epitopes: relationship to liver disease severity and alcohol consumption. Hepatology 25: 1418-1424.

22. Niemelä O, Israel Y. (1992) Hemoglobin-acetaldehyde adducts in human alcohol abusers. Lab. Invest. 67: 246-252.

23. Michot F, Gut J. (1987) Alcohol-induced bone marrow damage. A bone marrow study in alcohol-dependent individuals. Acta Haematol. 78: 252-257.

24. Meagher RC, Sieber F, Spivak JL. (1982) Suppression of hematopoietic-progenitor-cell proliferation by ethanol and acetaldehyde. N. Engl. J. Med. 30: 845-849.

25. Levine RF, Spivak JL, Meagher RC, Sieber F. (1986) Effect of ethanol on thrombosis. Br. J. Haematol. 62: 345-354.

26. Beauge F, Gallay J, Stibler H, Borg S. (1988) Alcohol abuse increases the lipid structural order in human erythrocyte membranes. A steady-state and time-resolved anisotrophy study. Biochem. Pharmacol. 37: 3823-3828.

27. Wickramasinghe SN, Malik F. (1986) Acetaldehyde causes a prolongation of the doubling time and an increase in the modal volume of cells in culture. Alcohol Clin. Exp. Res. 10: 350-354.

28. Wu A, Chanarin I, Levi AJ. (1974) Macrocytosis of chronic alcoholism. Lancet 4: 829-831.

29. Morgan MY, Camilo ME, Luck W, Sherlock S, Hoffbrand AV. (1981) Macrocytosis in alcohol-related liver disease: its value for screening. Clin. Lab. Haematol. 3: 35-44.

30. Savage DG, Ogundibe A, Allen RH, Stabler SP, Lindenbaum J. (2000) Etiology and diagnostic evaluation of macrocytosis. Am. J. Med. Sci. 319: 343-352.

31. Hazelett SE, Liebelt RA, Brown WJ, Androulakakis V, Jarjoura D, Truitt EB Jr. (1998) Evaluation of acetaldehydemodified hemoglobin and other markers of chronic heavy alcohol use: effects of gender and hemoglobin concentration. Alcohol Clin. Exp. Res. 22: 1811-1819.

32. Sarkola T, Eriksson CJP, Niemelä O, Sillanaukee P, Halmesmäki E. (2000) Mean cell volume and gamma-glutamyl transferase are superior to carbohydrate-deficient transferrin and hemoglobinacetaldehyde adducts in the follow-up of pregnant women with alcohol abuse. Acta Obstet. Gynecol. Scand. 79: 359-366.

33. Takeshita M, Morimoto K. (2000) Accumulation of hemoglobinassociated acetaldehyde with habitual alcohol drinking in the atypical ALDH2 genotype. Alcohol Clin. Exp. Res. 24: 1-7. 